\title{
Reliability of a dried urine test for comprehensive assessment of urine hormones and metabolites
}

\author{
Mark Newman ${ }^{*}$ and Desmond A. Curran
}

\begin{abstract}
Background: Mass spectrometry allows for analysis of multiple hormone and organic acid metabolites from small urine volumes; however, to assess the full extent of daily hormone production, 24-h urine collections are usually required. The aims of this study were, first, to confirm that mass spectrometric analysis of an array of hormones and organic acids would yield similar results in both liquid and dried urine, and, second, to determine if collection of four dried spot urine samples could be substituted for a 24-h collection when measuring reproductive hormones.
\end{abstract}

Methods: Two study populations were included in this prospective observational study. Twenty individuals collected both a spot liquid urine and dried urine on filter paper to analyze eight organic acids. A second group of 26 individuals collected both a 24-h urine and four dried spot urines during waking hours throughout the same day for evaluation of 17 reproductive hormones and metabolites; data from 18 of these individuals were available to compare liquid versus dried urine results. Dried urine was extracted, hydrolyzed, and derivatized before analysis by mass spectrometry; all analytes from dried urine were normalized to urine creatinine.

Results: Reproductive hormone results from dried and liquid urine were in excellent agreement with intraclass correlation coefficients (ICCS) greater than 0.90; comparison of dried to liquid urine for organic acids showed good to excellent agreement (ICC range: 0.75 to 0.99 ). Comparison between the 4-spot urine collection and 24-h urine collection methods showed excellent agreement (ICC >0.9) for 14 of the 17 urine metabolites and good agreement for the others (ICC 0.78 to 0.85 ) with no systematic differences between the two methods of collection.

Conclusions: The burden of urine collection can be reduced using collection of four spot dried urines on filter paper without compromising comparability with hormone results from a 24-h urine collection. A large number of urine analytes can be assessed from the dried urine with similar results to those from liquid urine. Given the ease of sample handling, this 4-spot dried urine assay would be useful for both clinical assessment of patients and for large epidemiologic studies.

Keywords: Dried urine testing, GC-MS/MS, LC-MS/MS, Reproductive hormones, Estrogen, Testosterone, Androgens, Organic acids

\begin{abstract}
Introduction
Analysis of an array of hormones or metabolites may be useful for the clinician faced with a patient with a multitude of nonspecific symptoms that lead to a large differential of diagnoses, for screening of multiple diseases at once, for a patient on hormonal replacement therapy $[1$,
\end{abstract}

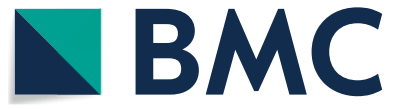

(c) The Author(s) 2021. This article is licensed under a Creative Commons Attribution 4.0 International License, which permits use, sharing, adaptation, distribution and reproduction in any medium or format, as long as you give appropriate credit to the original author(s) and the source, provide a link to the Creative Commons licence, and indicate if changes were made. The images or other third party material in this article are included in the article's Creative Commons licence, unless indicated otherwise in a credit line to the material. If material is not included in the article's Creative Commons licence and your intended use is not permitted by statutory regulation or exceeds the permitted use, you will need to obtain permission directly from the copyright holder. To view a copy of this licence, visit http://creativeco mmons.org/licenses/by/4.0/. The Creative Commons Public Domain Dedication waiver (http://creativecommons.org/publicdomain/ zero/1.0/) applies to the data made available in this article, unless otherwise stated in a credit line to the data. 
2], or for any patient where a complete picture of production and metabolism of a hormonal pathway is required [3]. Measurement of steroid hormones in urine can be an essential component to the diagnosis of hormone-related disorders $[4,5]$. Some patients may require sampling over multiple days to determine monthly variations or effects of change in treatment. For hormones with known circadian or pulsatile fluctuations, a representation of the entire day is essential [6]; however, collection and storage of a 24-h urine can be cumbersome for patients [7, 8].

Mass spectrometry technology, both liquid chromatography tandem mass spectrometry (LC-MS/MS) for water-soluble compounds and gas chromatography tandem mass spectrometry (GC-MS/MS) for non-polar compounds, is now routinely used to measure arrays of steroid hormones and organic acids because of its high assay sensitivity, accuracy with small volumes, and ability to evaluate multiple analytes at the same time $[9,10]$. This methodology allows for a complete profile of urine reproductive hormonal metabolites and multiple organic acids with high resolution of closely related structures [10].

Collection of urine on filter paper, which can then be dried and stored at room temperature until received by the laboratory [11-13], offers a significant advance in patient convenience and may improve patient adherence. If multiple samples are collected throughout the day, there is potential to capture both the diurnal variation of hormones along with the full range of daily hormonal production [11, 14]. Our prior proof-of-concept papers have demonstrated that dried urine leads to equivalent measures as liquid urine in our hands for cortisol, cortisone, and the cortisol metabolites, a-tetrahydrocortisol, $\beta$-tetrahydrocortisol, and tetrahydrocortisone [14] as well as for estrone, estradiol, a-pregnanediol, and $\beta$-pregnanediol [11]. Others have found similar results between liquid and dried urine with organic acids [12, 15, 16]. The urinary analytes in this study included female and male reproductive hormones, 6-hydroxymelatoninsulfate, and a number of organic acids, which have a multitude of uses (Additional file 1: Table S1). Inclusion of multiple metabolites from the estrogen and androgen pathways allows for a complete picture of estrogen and testosterone production and metabolism $[17,18]$.

The primary goal of this study was to confirm that measurement of the urinary profile of reproductive hormones, 6-hydroxymelatoninsulfate, and an array of organic acids extracted from dried urine collected on filter paper as analyzed by tandem mass spectrometry would provide results in agreement with measurements from liquid urine. The secondary aim was to demonstrate that measurement of reproductive hormones in a collection of four dried urine samples over a 15-h span throughout the day would accurately reflect the measurements of these hormones in a 24-h urine collection.

\section{Methods \\ Study populations}

A prospective observational study of urine collected from a population of healthy adult volunteers who agreed to participate in validation of urine analyses was conducted. This first study population included 26 individuals who provided data on hormonal measures (cortisol and cortisol metabolites, reproductive hormones, and 6-hydroxymelatoninsulfate) to compare samples from the 4-spot urine collection method to a 24-h urine collection. A subset of these individuals $(n=18)$ had data available to compare measures from dried versus liquid urine. As cortisol and cortisol metabolites [14] and $\alpha$ and $\beta$-pregnanediol [11] were validated in previous analyses, only the following hormones were included in this analysis: estrone (E1), estradiol (E2), estriol (E3), 2-hydroxyestrone (2OHE1), 2-hydroxyestradiol (2OHE2), 4-hydroxyestrone (4OHE1), 16-hydroxyestrone (16OHE1), 2-methoxyestrone (2-methoxyE1), testosterone (T), epitestosterone (EpiT), 5a- dihydrotestosterone (DHT), androsterone, etiocholanolone, 5a-androstanediol, $5 \beta$-androstanediol, dehydroepiandrosterone (DHEA), and 6-hydroxymelatoninsulfate. The data were collected between February and November of 2015 and informed consent was obtained from all participants.

The second analysis included 20 individuals whose deidentified data was pulled from the larger databank of 144,561 laboratory visits. Each of these samples included a single first-morning urine collection to compare results of dried versus liquid urine for the following organic acids: homovanillic acid (HVA), vanillylmandelic acid (VMA), kynurenic acid, xanthurenic acid, methylmalonic acid (MMA), pyroglutamic acid, 5-hydroxyindoleacetic acid (5-HIAA), and $\beta$-hydroxyisovaleric (Hiv) acid.

All volunteers in both study populations reported no medical problems and were not pregnant. Individuals were not excluded based on current or recent use of any hormonal medications, as the goal was only to compare measurement values for differing methodologies. Eighty percent of women in the first study population and all women in the second study population were premenopausal.

\section{Sample collection}

The 4-spot method involves urine samples collected at home at four times during the day: (1) the first urine of the day, (2) $2 \mathrm{~h}$ after awakening, (3) in the afternoon (approximately $4 \mathrm{PM}$ ), and 4) before bed (10PM). Participants collected samples by completely saturating $2 \times 3$ inches of filter paper (Whatman Body Fluid Collection 
Paper; Sigma-Aldrich, St. Louis, MO, USA) with urine. The paper was left exposed at room temperature for $24 \mathrm{~h}$ to dry. The stability of analytes in dried urine at room temperature for as long as 84 days has previously been demonstrated by this laboratory [11]. Dried samples were stored at $-80{ }^{\circ} \mathrm{C}$ until analyzed. Reproductive hormones were assessed in all four samples collected, while only the first morning sample was used for 6-hydroxymelatoninsulfate and the organic acid tests.

During the same day, all liquid urine samples for the 24-h collection were added to a low-density polyethylene plastic container (ES Robbins, Muscle Shoals, AL, USA) container with approximately $1 \mathrm{~g}$ of boric acid (SigmaAldrich, St. Louis, MO, USA) and kept refrigerated for the duration of the collection. The four dried urine samples removed a total of about $8 \mathrm{~mL}$ of urine from the 24-h collection. This was considered negligible and was not accounted for. The total volume of urine from 24-h collections was measured, and an aliquot was frozen and stored at $-80^{\circ} \mathrm{C}$ until tested.

\section{Urine reproductive hormone analysis}

The urinary steroid hormones were analyzed using proprietary in-house CLIA (Clinical Laboratory Improvement Amendments) approved assays on the Agilent 7890/7000B GC-MS/MS (Agilent Technologies, Santa Clara, CA, USA). All reagents, unless otherwise noted, were purchased from Sigma-Aldrich (St. Louis, MO, USA). A $600 \mathrm{ul}$ aliquot of liquid urine was taken from the sample collection and the equivalent of approximately $600 \mathrm{ul}$ of urine was extracted from the filter paper using $2 \mathrm{~mL}$ of $100 \mathrm{mM}$ ammonium acetate adjusted to a $\mathrm{pH}$ of 5.9. These aliquots of the conjugated hormones were transferred to a C18 solid phase extraction (SPE) column (UCT LLC, Briston, PA, USA), eluted using methanol, and the eluate was dried under nitrogen at $40^{\circ} \mathrm{C}$.

The conjugated hormones were then hydrolyzed from their glucuronide and sulfate forms to free forms using enzymes from Helix pomatia (Sigma-Aldrich, St. Louis, MO, USA) in acetate buffer $\left(55{ }^{\circ} \mathrm{C}, 90 \mathrm{~min}\right)$. The enzymatic reaction was quenched with sodium hydroxide and the hormones extracted with ethyl acetate. The ethyl acetate extracts were dried under nitrogen at $40{ }^{\circ} \mathrm{C}$. The analytes were derivatized using a mixture of $100 \mathrm{ul}$ acetonitrile $(\mathrm{ACN})$ and $50 \mathrm{ul} \mathrm{bis(trimethylsilyl)trifluoroacet-}$ amide (Sigma-Aldrich, St. Louis, MO, USA) for $30 \mathrm{~min}$ at $70{ }^{\circ} \mathrm{C}$. Internal standards (Steraloids, Newport, RI, USA) were added prior to ethyl acetate extraction, and the percentage recovery from all assays was greater than 90\%. Derivatized extract (1.6 ul) was injected into the GC-MS/MS. Samples and controls were analyzed along with a standard curve spanning the expected range of concentrations. Instrument conditions for the oven were an initial temperature of $130{ }^{\circ} \mathrm{C}$ increasing to $200{ }^{\circ} \mathrm{C}$ at $25{ }^{\circ} \mathrm{C} / \mathrm{min}$, then to $230{ }^{\circ} \mathrm{C}$ at $4.3{ }^{\circ} \mathrm{C} / \mathrm{min}$, and finally to $290{ }^{\circ} \mathrm{C}$ at $25{ }^{\circ} \mathrm{C} / \mathrm{min}$. Multiple reaction monitoring transitions for ion mass $>$ ion product of fragmentation can be found in Table 1. Creatinine was measured using a conventional colorimetric (Jaffe) method, after initial extraction from the filter paper. The average inter-assay coefficient of variation was $6.7 \%$ for creatinine. In addition to expressing the measures per mg of creatinine to correct for variations in filter paper saturation and hydration status, a secondary equation was applied to reduce bias related to the effects of age, sex, weight, and height on creatinine excretion [19].

Urine 6-hydroxymelatoninsulfate and organic acid analysis The hydrophilic analytes were assessed by LC-MS/MS using proprietary in-house CLIA approved assays. For the 6-hydroxymelatoninsulfate assay, a $30 \mathrm{ul}$ aliquot was taken from the methanol elution of both the liquid urine collection and the waking sample dried urine collected on filter paper. This extract was then reconstituted in 130 ul of deionized water. For the organic acids, a $100 \mathrm{ul}$ aliquot of liquid urine was taken and an equivalent amount was extracted from the waking sample dried urine filter paper using $250 \mathrm{ul}$ of water with the addition of $50 \mathrm{ul}$ $100 \mathrm{mM}$ ammonium acetate (Sigma-Aldrich, St. Louis, MO, USA) adjusted to a $\mathrm{pH}$ of 5.9 and $2 \%$ formic acid.

For 6-hydroxymelatoninsulfate, $20 \mathrm{ul}$ was injected into an ultra-performance liquid chromatography (UPLC) (Waters Corporation, Milford, MA, USA) column with a

Table 1 Multiple reaction monitoring transitions for ion mass $>$ ion product of fragmentation for urinary reproductive hormones

\begin{tabular}{ll}
\hline Analyte & Transition \\
\hline Estrone (E1) & $342.1>257.1$ \\
Estradiol (E2) & $416.2>285.1$ \\
Estriol (E3) & $504.3>296.3$ \\
2-Hydroxyestrone (2OHE1) & $430.2>345.0$ \\
2-Hydroxyestradiol (2OHE2) & $462.2 .2>195.2$ \\
4-Hydoxyesrone (4OHE1) & $430.2>354.0$ \\
16-Hydroxyestrone (16OHE1) & $430.2>286.1$ \\
2-Methoxyestrone (2-methoxyE1) & $372.2-342.1$ \\
Testosterone (T) & $360.2>174.1$ \\
Epitestosterone (EpiT) & $360.2>174.1$ \\
Dihydrotestosterone (DHT) & $347.2>271.2$ \\
Androsterone & $347.2>271.2$ \\
Etiocholanolone & $347.2>253$ \\
5a-androstanediol & $421.2>331.2$ \\
5 3 -androstanediol & $241.2>185.2$ \\
Dehydroepiandrosterone (DHEA) & $303.7>199.1$ \\
\hline
\end{tabular}


Waters ${ }^{\mathrm{TM}}$ tandem quadrupole mass spectrometer detector (TQD). The sample was eluted from a $1.8 \mathrm{u} 2.1 \times 50 \mathrm{~mm}$ pentafluorophenyl (PFP) column (Agilent Technologies, Santa Clara, CA, USA) using a gradient of $95 \% 0.001 \%$ formic acid in 5\% ACN to $45 \% 0.001 \%$ formic acid in 55\% $\mathrm{ACN}$. For the organic acids, $5 \mathrm{ul}$ was injected into the Waters ${ }^{\mathrm{TM}}$ UPLC column with TQD. These analytes were eluted from a 1.6 um $2.1 \times 50 \mathrm{~mm}$ Luna Omega PS C18 column (Phenomenex, Torrance, CA, USA) using a gradient $99.9 \% 0.2 \%$ formic acid in $0.1 \% \mathrm{ACN}$ to $73 \% 0.2 \%$ formic acid in $27 \%$ ACN. Multiple reaction monitoring transitions for ion mass > ion product of fragmentation for urine 6-hydroxymelatoninsulfate and the organic acids are listed in Table 2. The same creatinine corrections of the measures used for the reproductive hormones were also used for 6-hydroxymelatoninsulfate and the organic acids.

\section{Statistical methods}

A sample size of 18 individuals provides a power of greater than $80 \%$ to detect an intraclass correlation coefficient (ICC) of at least 0.6 with an alpha of 0.05 [20]. The statistical analyses were performed using SAS/STAT ${ }^{\circledR}$ software, Version 9.3 (SAS Institute Inc., Cary, NC, USA) and generated 2-sided p-values.

Variables are described as mean \pm standard deviation if normally distributed and median (interquartile range (IQR)) if the distribution was skewed. Student's $t$ test (for normally distributed variables) or the Wilcoxon rank-sum test (for skewed variables) were used to determine differences between men and women. Spearman

Table 2 Multiple reaction monitoring transitions for ion mass $>$ ion product of fragmentation for urine 6-hydroxymelatoninsulfate and the organic acids

\begin{tabular}{ll}
\hline Analyte & Transition \\
\hline 6-Hydroxymelatoninsulfate & $372.5>176.2$ \\
d4-6-Hydroxymelatoninsulfate & $331.5>179.2$ \\
Homovanillic acid (HVA) & $183.0>137.0$ \\
d2-Homovanillic acid (d2-HVA) & $185.0>139.0$ \\
Vanillylmandelic acid (VMA) & $197.0>138.0$ \\
d3-Vanillylmandelic acid (d3-VMA) & $199.8>140.9$ \\
Kynurenic acid & $190.0>144.0$ \\
d5-Kynurenic acid & $192.0>149.0$ \\
Xanthurenic acid & $206.0>160.0$ \\
d3-Xanthurenic acid & $210.0>164.0$ \\
Methylmalonic acid (MMA) & $117.0>73.0$ \\
d2-Methylmalonic acid (d2-MMA) & $120.0>76.0$ \\
Pyroglutamic acid & $130.0>84.0$ \\
d5-Pyroglutamic acid & $135.0>89.0$ \\
5-Hydroxyindoleacetic acid (5-HIAA) & $192.0>146.0$ \\
\hline
\end{tabular}

correlation coefficients $(\rho)$ were used to determine interclass associations between variables.

Consistency between liquid versus dried urine measures and 4-spot versus 24-h collection methodology was assessed using intraclass correlation coefficients (ICC). ICCs, which range from 0 to 1 with proximity to 1 indicating better agreement, assess for agreement of a measure between two differing methodologies within individuals [21]. Skewed variables were log transformed to approximate a normal distribution prior to assessing ICCs. As 4-spot (ng/mg-Cr) and 24-h (ug/d) measures were expressed in differing units, sex-specific Z-scores ([individual measurement-mean]/standard deviation) were created to standardize the measures and allow for direct comparison. Comparisons of differences between measures within an individual were assessed using signed-rank tests (for skewed variables) or paired t-tests (for normally distributed variables). Because the hypotheses of this paper were intrinsically correlated, no adjustments were made for multiple comparisons.

\section{Results}

\section{Study populations}

Characteristics of the first study population $(n=26)$ are shown in Table 3 . All of these individuals (58\% female; $100 \%$ Caucasian) had data available for comparison of 4-spot versus 24-h urine samples for male and female reproductive hormones. A subset (10 female, 8 male) also had measurements to compare liquid versus dried urine samples for both the reproductive hormones and 6-hydroxymelatoninsulfate. Characteristics of the second study population $(\mathrm{n}=20 ; 75 \%$ female; $80 \%$ Caucasian $/ 10 \%$ Hispanic/10\% Asian-Pacific Islander), data from whom were used to compare a single first morning collection of liquid versus dried urine samples for the organic acid tests, are provided in Table 4.

\section{Agreement between Liquid and Dried Urine Measures}

For the majority of analytes, there was excellent agreement (ICC $>=0.90$ ) between the liquid and dried measures (Table 5). The exceptions were VMA $(\mathrm{ICC}=0.79)$ and pyroglutamic acid $(\mathrm{ICC}=0.75)$, which still had good agreement. Similarly, for the majority of analytes, there was no systemic directionality to the difference in the dried urine compared to the liquid urine. However, estrone and estriol were consistently higher when measured in liquid urine, while some of the organic acids - VMA, kynurenic acid, pyroglutamic acid, and $\beta$-hydroxyisovaleric acid - were more concentrated in the dried urine sample (Table 5). Representative interclass correlations (Spearman) between the two methods are shown in Fig. 1 and Fig. 2 (the rest are available in Additional file 1: Figure S1). 
Table 3 Age and 24-h measures of reproductive hormones and 6-hydroxymelatoninsulfate of the first study population

\begin{tabular}{|c|c|c|c|}
\hline Variable & All & Female $(n=15)$ & Male $(n=11)$ \\
\hline Age (years) & $36.8 \pm 14.5$ & $33.7 \pm 7.8$ & $37.8 \pm 18$ \\
\hline Estrone $(\mathrm{ug} / \mathrm{d})^{\mathrm{a}}$ & $13.69(6.32,19.16)$ & $18.75(6.83,23.68)$ & $7.67(6.26,13.68)$ \\
\hline Estradiol (ug/d) & $1.73(0.80,3.26)$ & $3.17(0.89,4.19)$ & $1.14(0.70,1.62)$ \\
\hline Estriol (ug/d) $)^{a}$ & $6.82(3.57,12.80)$ & $11.64(6.14,16.55)$ & $5.56(3.45,6.91)$ \\
\hline 2-Hydroxyestrone (ug/d) ${ }^{a}$ & $3.33(1.27,6.24)$ & $5.82(0.77,8.46)$ & $2.26(1.27,4.03)$ \\
\hline 2-Hydroxyestradiol (ug/d) ${ }^{\mathrm{a}}$ & $0.25(0.11,0.67)$ & $0.58(0.06,0.81)$ & $0.19(0.11,0.26)$ \\
\hline 4-Hydroxyestrone (ug/d) ${ }^{\mathrm{a}}$ & $0.37(0.19,0.75)$ & $0.67(0.19,0.99)$ & $0.30(0.16,0.36)$ \\
\hline 16-Hydroxyestrone (ug/d) ${ }^{a}$ & $0.89(0.42,1.48)$ & $1.40(0.36,1.58)$ & $0.49(0.42,0.90)$ \\
\hline 2-Methoxyestrone $(\mathrm{ug} / \mathrm{d})^{\mathrm{a}}$ & $2.27(1.30,3.80)$ & $3.56(1.38,5.50)$ & $1.63(1.05,2.35)$ \\
\hline Testosterone $(\mathrm{ug} / \mathrm{d})^{\mathrm{b}}$ & $9.15(4.97,51.92)$ & $5.43(4.51,8.01)$ & $61.40(42.14,97.70)$ \\
\hline Epitestosterone $(\mathrm{ug} / \mathrm{d})^{\mathrm{b}}$ & $7.23(4.01,11.23)$ & $4.44(1.18,7.49)$ & $14.94(7.99,16.80)$ \\
\hline 5a-Dihydrotestosterone $(\mathrm{ug} / \mathrm{d})^{\mathrm{b}}$ & $5.53(2.72,11.59)$ & $2.72(1.33,5.46)$ & $12.60(9.88,17.47)$ \\
\hline Androsterone $(\mathrm{ug} / \mathrm{d})^{\mathrm{a}}$ & $1331.99(854.41,1940.29)$ & $1032.77(691.70,1528.59)$ & $2083.52(1005.51,2716.33)$ \\
\hline Etiocholanolone (ug/d) ${ }^{\mathrm{a}}$ & $811.74(463.17,1045.23)$ & $568.56(430.73,853.81)$ & $1020.79(716.22,1423.92)$ \\
\hline 5a-Androstanediol (ug/d) ${ }^{b}$ & $102.50(54.83,197.14)$ & $58.72(46.49,97.50)$ & $229.01(143.30,292.53)$ \\
\hline $5 \beta$-Androstanediol $(\mathrm{ug} / \mathrm{d})^{\mathrm{a}}$ & $35.58(14.05,60.75)$ & $15.83(9.38,52.69)$ & $60.75(20.69,122.18)$ \\
\hline DHEA (ug/d) & $166.14(80.45,431.61)$ & $136.42(80.45,317.83$ & $351.32(52.69,732.63)$ \\
\hline 6-Hydroxymelatoninsulfate (ug/ml) & $7.87(5.93,13.85)$ & $7.48(5.93,8.43)$ & $12.21(5.11,20.06)$ \\
\hline
\end{tabular}

Age presented as mean $\pm S D$; hormone data presented as median (IQR) as these data were skewed

a $\mathrm{p}<0.05 ;{ }^{\mathrm{b}} \mathrm{p}<0.01$ for differences between males and females as assessed by the Wilcoxon rank-sum test

DHEA: dehydroepiandrosterone

Table 4 Age and measures of organic acids from an early morning spot urine collection of the second study population

\begin{tabular}{llll}
\hline Variable & All & Female $(n=15)$ & Male $(n=5)$ \\
\hline Age (years) & $34.4 \pm 6.6$ & $34.5 \pm 6.6$ & $34.0 \pm 7.4$ \\
Methylmalonic acid $(\mathrm{ng} / \mathrm{mg}-\mathrm{Cr})$ & $0.18(0.16,0.25)$ & $0.20(0.16,0.29)$ & $0.16(0.16,0.18)$ \\
${\text { Homovanillic acid }(\mathrm{ng} / \mathrm{mg}-\mathrm{Cr})^{\mathrm{b}}}_{\text {Vanillylmandelic acid }(\mathrm{ng} / \mathrm{mg}-\mathrm{Cr})}$ & $0.52 \pm 0.16$ & $0.57 \pm 0.14$ & $0.35 \pm 0.05$ \\
Kynurenic acid $(\mathrm{ng} / \mathrm{mg}-\mathrm{Cr})$ & $0.41 \pm 0.12$ & $0.43 \pm 0.12$ & $0.36 \pm 0.13$ \\
Xanthurenic acid $(\mathrm{ng} / \mathrm{mg}-\mathrm{Cr})$ & $0.25 \pm 0.08$ & $0.26 \pm 0.08$ & $0.21 \pm 0.05$ \\
5-HIAA (ng/mg-Cr) & $0.06(0.05,0.07)$ & $0.06(0.04,0.07)$ & $0.06(0.05,0.07)$ \\
Pyroglutamic acid $(\mathrm{ng} / \mathrm{mg}-\mathrm{Cr})$ & $0.44(0.40,0.55)$ & $0.47(0.41,0.58)$ & $0.37(0.36,0.41)$ \\
$\beta$-Hydroxyisovaleric acid $(\mathrm{ng} / \mathrm{mg}-\mathrm{Cr})$ & $4.06 \pm 0.69$ & $4.11 \pm 0.78$ & $3.90 \pm 0.33$ \\
\hline
\end{tabular}

Data are presented as mean $\pm S D$ if normally distributed and median (IQR) if right-skewed

${ }^{a} p<0.05 ;{ }^{b} p<0.01$ for differences between males and females as assessed by the Student t-test or Wilcoxon rank-sum test

5-HIAA: 5-hydroxyindoleacetic acid

\section{Agreement between the DUTCH 4-spot and 24-h urine collection for hormonal measures}

The measurement of reproductive hormones in urine samples collected four times throughout the day were comparable to the gold standard of a 24-h urine collection (Table 6). As the measures from the 4-spot urine collection are reported in $\mathrm{ng} / \mathrm{mg}-\mathrm{Cr}$ and the 24-h urine measures are reported in $\mathrm{ug} / \mathrm{d}$, sex-specific Z-scores were created for direct comparison of the two methodologies. There was excellent consistency $(\mathrm{ICC}>0.9)$ for the majority of the analytes and good consistency for the remainder (estriol, $5 a$ and $5 \beta$-androstanediol) (Table 6). There was no systematic directionality to the differences between any of the Z-scores (Table 6). Representative interclass (Spearman) correlations between the analytes are shown in Fig. 3 (the remainder are shown in Additional file 1: Figure S3).

A sensitivity analysis to verify the need for creatinine correction was done by calculating ICCs for the agreement between sex-specific Z-scores from the 24-h 
Table 5 Comparison of liquid versus dried urine analytes $(n=18)$

\begin{tabular}{|c|c|c|c|c|}
\hline Variable & Dried & Liquid & Difference $(95 \% \mathrm{Cl})$ & ICC (95\% Cl) \\
\hline \multicolumn{5}{|l|}{ Hormones $(n=18)$} \\
\hline Estrone (ug/d) & $10.78(6.26,18.73)$ & $12.78(7.22,20.65)$ & $1.28[0.09,2.47]^{\mathrm{a}}$ & $0.92[0.80,0.97]$ \\
\hline Estradiol (ug/d) & $1.32(0.70,3.26)$ & $1.29(0.74,3.36)$ & $0.09[-0.01,0.19]$ & $0.96[0.89,0.98]$ \\
\hline Estriol (ug/d) & $6.82(3.45,11.64)$ & $7.15(4.76,14.41)$ & $0.86[0.05,1.67]^{a}$ & $0.90[0.77,0.96]$ \\
\hline 2-Hydroxyestrone (ug/d) & $2.22(0.98,6.24)$ & $1.84(1.17,6.24)$ & $-0.01[-0.18,0.16]$ & $0.98[0.94,0.99]$ \\
\hline 2-Hydroxyestradiol (ug/d) & $0.18(0.09,0.58)$ & $0.18(0.08,0.47)$ & $0.00[-0.02,0.03]$ & $0.97[0.92,0.99]$ \\
\hline 4-Hydroxyestrone (ug/d) & $0.31(0.16,0.67)$ & $0.40(0.25,0.62)$ & $0.03[-0.02,0.09]$ & $0.95[0.87,0.98]$ \\
\hline 16-Hydroxyestrone (ug/d) & $0.69(0.42,1.48)$ & $0.61(0.39,1.24)$ & $-0.07[-0.18,0.05]$ & $0.95[0.87,0.98]$ \\
\hline 2-Methoxyestrone (ug/d) & $1.64(1.05,3.56)$ & $1.70(1.50,3.58)$ & $0.12[-0.07,0.30]$ & $0.97[0.94,0.99]$ \\
\hline Testosterone (ug/d) & $7.64(4.97,49.46)$ & $8.42(5.80,51.40)$ & $2.07[-0.26,4.40]$ & $0.99[0.98,1.00]$ \\
\hline Epitestosterone (ug/d) & $7.23(4.01,11.23)$ & $8.21(4.23,13.98)$ & $0.51[-0.06,1.07]$ & $0.99[0.97,1.00]$ \\
\hline 5a-Dihydrotestosterone (ug/d) & $5.53(2.72,11.59)$ & $4.94(2.68,10.35)$ & $-0.61[-1.02,-0.19]^{\mathrm{a}}$ & $0.99[0.97,1.00]$ \\
\hline Androsterone (ug/d) & $1331.99(929.43,1933.30)$ & $1237.63(813.31,1847.10)$ & $-33.02[-105.70,39.67]$ & $0.99[0.98,1.00]$ \\
\hline Etiocholanolone (ug/d) & $847.93(430.73,1088.18)$ & $943.49(414.13,1161.61)$ & $19.76[-13.02,52.54]$ & $0.99[0.98,1.00]$ \\
\hline 5a-Androstanediol (ug/d) & $110.09(57.50,197.14)$ & $119.40(65.63,226.93)$ & $15.67[-12.67,44.01]$ & $0.90[0.76,0.96]$ \\
\hline $5 \beta$-Androstanediol (ug/d) & $35.58(14.05,72.42)$ & $46.39(14.72,65.39)$ & $-0.54[-13.93,12.85]$ & $0.88[0.73,0.95]$ \\
\hline DHEA (ug/d) & $131.42(52.69,444.35)$ & $130.86(53.27,457.05)$ & $8.65[-36.66,53.96]$ & $1.00[0.99,1.00]$ \\
\hline 6-Hydroxymelatoninsulfate (ug/d) & $7.87(5.93,14.33)$ & $8.26(5.93,14.67)$ & $0.04[-1.14,1.21]$ & $0.99[0.98,1.00]$ \\
\hline \multicolumn{5}{|l|}{ Organic Acids $(n=20)$} \\
\hline Methylmalonic acid (ng/mg-Cr) & $0.18(0.15,0.23)$ & $0.18(0.16,0.25)$ & $0.00[-0.01,0.02]$ & $0.96[0.90,0.98]$ \\
\hline Homovanillic acid (ng/mg-Cr) & $0.52 \pm 0.17$ & $0.52 \pm 0.16$ & $-0.01[-0.05,0.02]$ & $0.88[0.74,0.95]$ \\
\hline Vanillylmandelic acid (ng/mg-Cr) & $0.45 \pm 0.13$ & $0.41 \pm 0.12$ & $-0.05[-0.08,-0.02]^{\mathrm{a}}$ & $0.79[0.57,0.91]$ \\
\hline Kynurenic acid (ng/mg-Cr) & $0.26 \pm 0.08$ & $0.25 \pm 0.08$ & $-0.01[-0.02,-0.00]^{\mathrm{a}}$ & $0.95[0.88,0.98]$ \\
\hline Xanthurenic acid (ng/mg-Cr) & $0.06(0.05,0.08)$ & $0.06(0.05,0.07)$ & $-0.00[-0.00,0.00]$ & $0.98[0.95,0.99]$ \\
\hline 5-HIAA (ng/mg-Cr) & $0.46(0.43,0.57)$ & $0.44(0.40,0.55)$ & $-0.01[-0.05,0.02]$ & $0.98[0.96,0.99]$ \\
\hline Pyroglutamic acid (ng/mg-Cr) & $4.42 \pm 0.72$ & $4.06 \pm 0.69$ & $-0.41[-0.58,-0.23]^{b}$ & $0.75[0.51,0.89]$ \\
\hline$\beta$-Hydroxyisovaleric acid (ng/mg-Cr) & $0.29 \pm 0.12$ & $0.27 \pm 0.12$ & $-0.02[-0.03,-0.00]^{\mathrm{a}}$ & $0.95[0.88,0.98]$ \\
\hline
\end{tabular}

For liquid and dried measurements, data presented as mean \pm SD for normally distributed variables and median (IQR) for skewed variables; the differences were normally distributed and are presented as mean $[95 \% \mathrm{CI}]$

${ }^{a} \mathrm{p}<0.05 ;{ }^{\mathrm{b}} \mathrm{p}<0.0001$ for the liquid minus dried difference by paired t-test

5-HIAA: 5-hydroxyindoleacetic acid; Cr: creatinine, DHEA: dehydroepiandrosterone; ICC: intraclass correlation coefficient

measures and the 4-spot assay without correction for creatinine. Without the creatinine correction, the ICCs were all lower than those observed with the creatinine correction (with the exception of estriol which had a slightly higher ICC by 0.04 ) with the degree of difference ranging from -0.04 to 0.15 and averaging 0.07 . An example of the interclass correlations (Spearman) between the 24-h measures and the 4-spot assay with and without the creatinine corrections is shown in Additional file 1: Figure S4.

\section{Discussion}

This study demonstrated the feasibility of accurately measuring multiple (up to 32) analytes in dried urine samples collected on filter paper using assays that conform to CLIA criteria. All measurements from dried urine demonstrated at least good agreement with measures from liquid urine, and the majority (83\%) demonstrated excellent agreement with intraclass correlations greater than 0.9. For most analytes, neither loss nor excess concentration occurred during the sample drying or laboratory extraction process. In addition, measurement of the reproductive steroid hormones, which are usually evaluated from a 24-h collection due to their pulsatile release [22], were well represented by the 4-spot dried urine collection with at least good agreement of the 4-spot measurement with the 24-h gold standard measure for all steroid metabolites and excellent agreement $(>0.9)$ for the majority (82\%). There were no systematic differences between the relative amount of hormone collected by either methodology. The 4-spot dried urine (DUTCH) methodology allows for efficient, accurate assessment of numerous urine metabolites using a convenient collection method, while avoiding the need for a full 24-h liquid urine collection. 

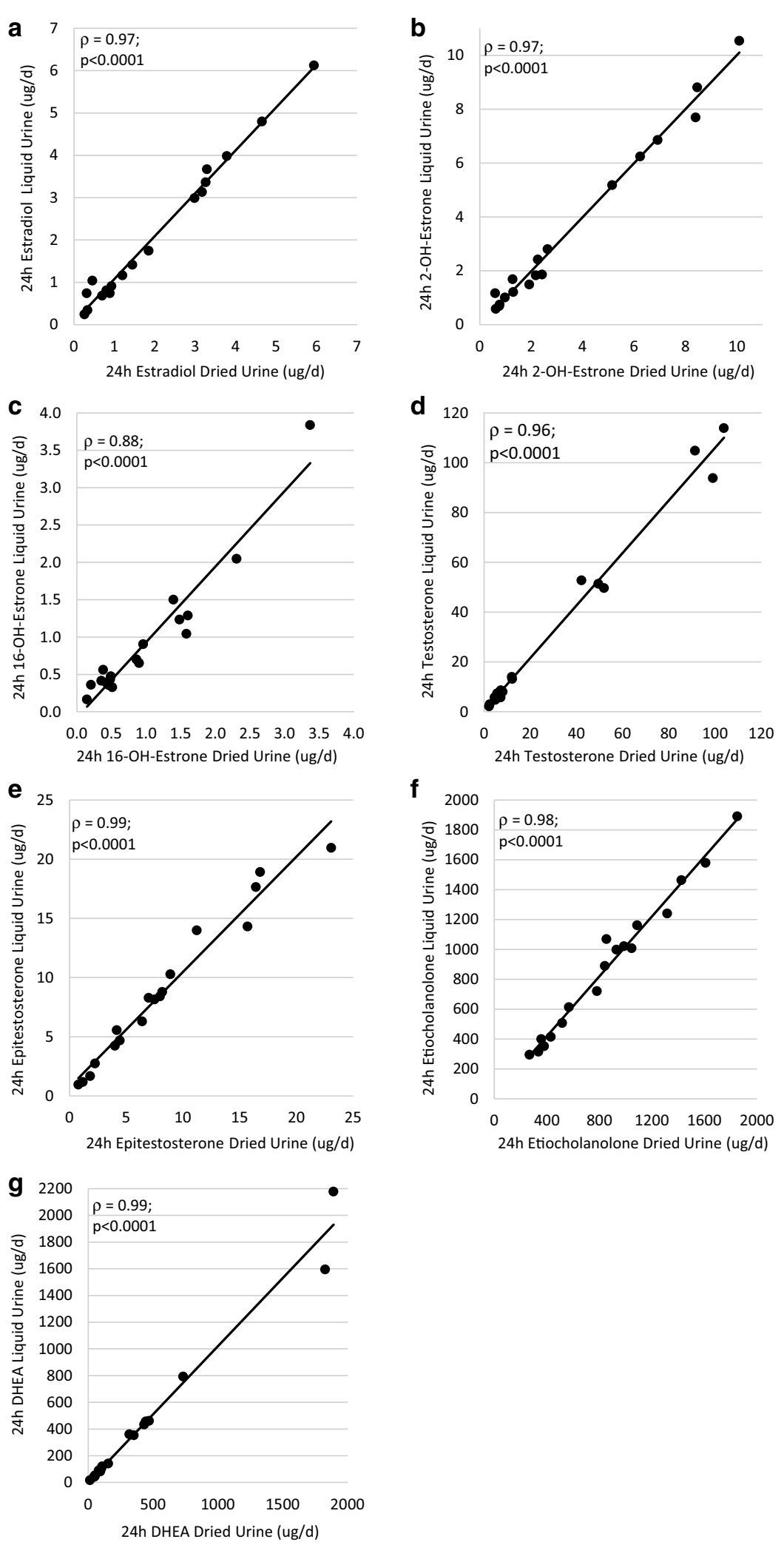

Fig. 1 Correlations between the liquid versus dried measurements for select urine steroid hormones. The remainder are available in Additional file 1: Figure S1. Reported correlation coefficients are Spearman correlations. Cr: creatinine; DHEA: dehydroepiandrosterone 

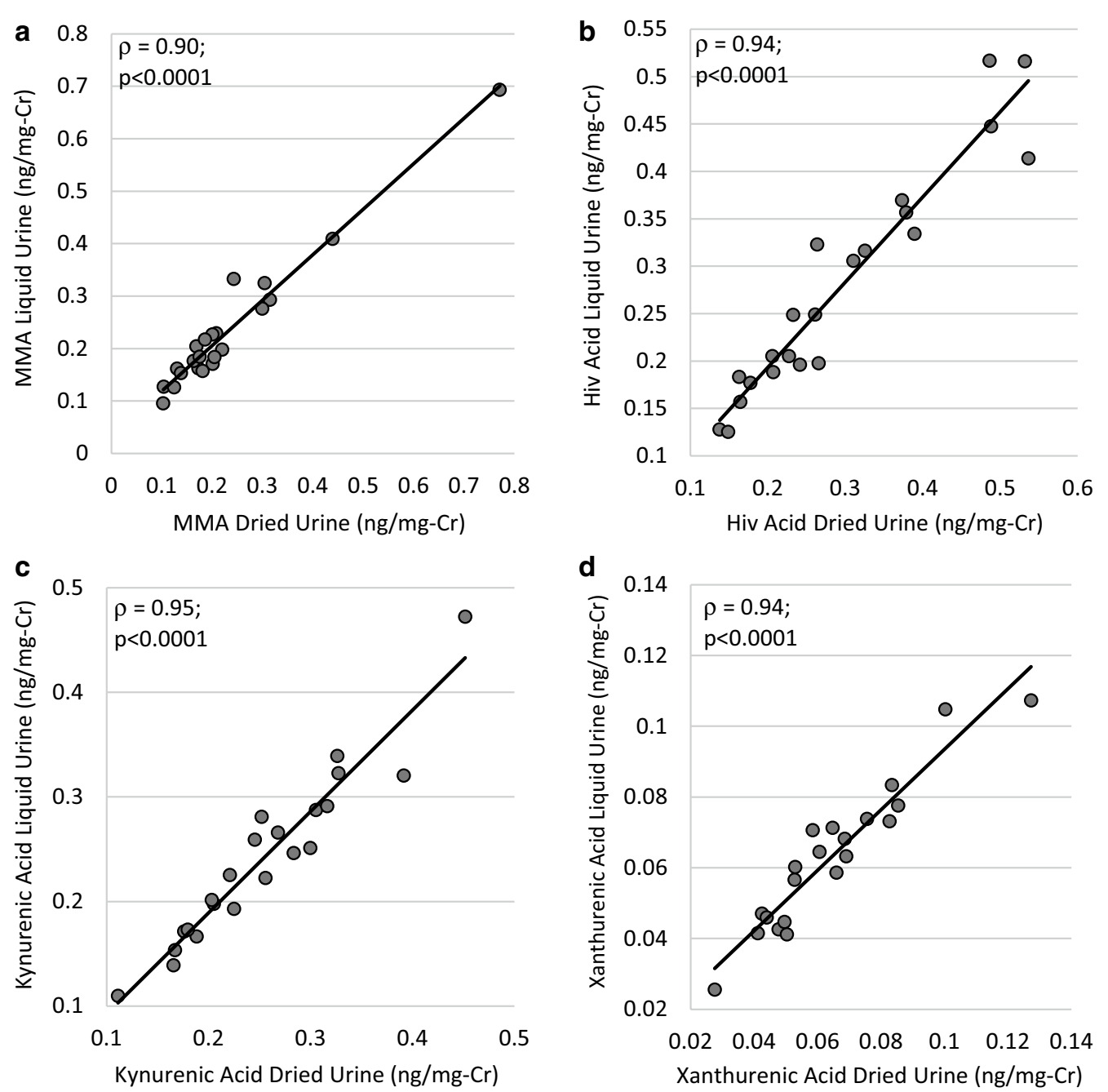

Fig. 2 Correlations between the liquid versus dried measurements for select urine organic acids. The remainder are available in Additional file 1: Figure S2. Reported correlation coefficients are Spearman correlations. Cr: creatinine, Hiv: $\beta$-Hydroxyisovaleric

The 4-spot dried urine collection has previously been shown to be representative of select 24-h measures of steroidal hormones by our group [11], as measurement of urinary a-pregnanediol, $\beta$-pregnanediol, estrone, and estradiol with this method are representative of both 24-h urine collections as well as serum hormone concentrations. In fact, repeated assessments over a month demonstrated that the dried urine collections could accurately recreate the changes observed in serum during the menstrual cycle [11]. In addition, not only does the 4-spot urine method accurately represent a 24-h urine collection for urinary cortisol, cortisone, and cortisol metabolites, but it can also be used to represent the expected diurnal pattern observed with salivary measures, if each of the four collections is considered individually [14].
Previous studies have validated the usefulness of high throughput GC-MS/MS for urine steroid profiling of more than 30 metabolites; however, this was done in liquid urine [5]. Conversely, measurement of organic acids in spot dried urine samples using filter paper has already been well-validated as a technique for screening neonates for metabolic disorders [15, 23, 24] and for screening for neuroblastoma [25] with similar recoveries obtained from liquid and dried urine. Dried urine has also been used to measure other urine analytes of interest, such as sodium and potassium, with similarly high levels of stability [26]. As with others who used either filter paper or cotton swabs [27], we found that dried urine results are in agreement with liquid urine results, reduced the 
Table 6 Comparison of the urinary hormone profile using the 4-spot (DUTCH) or 24-h urine collection method $(n=26)$

\begin{tabular}{lllll}
\hline Variable & 24-h urine collection $(\mathrm{ug} / \mathrm{d})$ & 4-spot $(\mathrm{ng} / \mathrm{mg}-\mathrm{Cr})$ & Z-score difference $[95 \% \mathrm{Cl}]^{\mathrm{a}}$ & $\mathrm{ICC}[95 \% \mathrm{Cl}]$ \\
\hline Estrone & $13.69(6.32,19.16)$ & $14.37(7.13,18.75$ & $0.00[-0.13,0.13]$ & $0.95[0.89,0.97]$ \\
Estradiol & $1.73(0.80,3.26)$ & $1.68(0.92,3.81)$ & $0.00[-0.16,0.16]$ & $0.92[0.83,0.96]$ \\
Estriol & $6.82(3.57,12.80)$ & $7.39(4.15,12.96)$ & $0.00[-0.24,0.24]$ & $0.82[0.64,0.91]$ \\
2-Hydroxyestrone & $3.33(1.27,6.24)$ & $3.72(1.45,6.75)$ & $-0.00[-0.09,0.09]$ & $0.97[0.94,0.99]$ \\
2-Hydroxyestradiol & $0.25(0.11,0.67)$ & $0.30(0.13,0.49)$ & $-0.00[-0.10,0.10]$ & $0.97[0.93,0.99]$ \\
4-Hydroxyestrone & $0.37(0.19,0.75)$ & $0.45(0.27,0.81)$ & $-0.00[-0.15,0.15]$ & $0.93[0.86,0.97]$ \\
16-Hydroxyestrone & $0.89(0.42,1.48)$ & $0.93(0.57,1.69)$ & $0.00[-0.19,0.19]$ & $0.90[0.77,0.95]$ \\
2-Methoxyestrone & $2.27(1.30,3.80)$ & $2.11(1.54,4.15)$ & $0.00[-0.19,0.19]$ & $0.90[0.78,0.95]$ \\
Testosterone & $9.15(4.97,51.92)$ & $9.33(5.36,54.73)$ & $-0.00[-0.10,0.10]$ & $0.97[0.93,0.98]$ \\
Epitestosterone & $7.23(4.01,11.23)$ & $7.53(3.81,13.90)$ & $-0.00[-0.07,0.07]$ & $0.99[0.97,0.99]$ \\
5a-Dihydrotestosterone & $5.53(2.72,11.59)$ & $6.40(2.16,11.91)$ & $-0.00[-0.09,0.09]$ & $0.97[0.94,0.99]$ \\
Androsterone & $1331.99(854.41,1940.29)$ & $1346.46(818.59,2065.66)$ & $-0.00[-0.09,0.09]$ & $0.97[0.94,0.99]$ \\
Etiocholanolone & $811.74(463.17,1045.23)$ & $797.60(475.43,1061.66)$ & $-0.00[-0.09,0.09]$ & $0.98[0.95,0.99]$ \\
5a-Androstanediol & $102.50(54.83,197.14)$ & $86.74(51.31,200.70)$ & $-0.00[-0.27,0.27]$ & $0.78[0.57,0.89]$ \\
53-Androstanediol & $35.58(14.05,60.75)$ & $34.49(13.91,62.77)$ & $0.00[-0.22,0.22]$ & $0.85[0.70,0.93]$ \\
DHEA & $166.14(80.45,431.61)$ & $162.85(78.17,394.62)$ & $-0.00[-0.05,0.05]$ & $0.99[0.99,1.00]$ \\
\hline
\end{tabular}

The ICCs were calculated between measurements expressed as standardized sex-specific Z-scores (mean \pm SD for all $=0.00 \pm 0.98)$. Data are presented as median (IQR) for measurements and the difference (24-h minus 4-spot) in individual Z-scores is presented as mean [95\% confidence interval]

a All p-values $>0.9$ by paired t-test

Cr: creatinine; DHEA: dehydroepiandrosterone; ICC: intraclass correlation coefficient

burden on patients, and had good stability over time [11]. Still, agreement between 24-h urine collections and early-morning, single spot urine collections for hormonal analysis are often poor [28]. This study now extends our prior findings $[11,14]$ to show that the increase to four spot urines spaced throughout the waking hours provides better coverage of the hormonal output for all the male and female reproductive hormones and metabolites, resulting in strong agreement with 24-h urine measures.

There are some caveats that must be considered when interpreting our results. During the urine collection, there may have been differences in saturation of the filter paper. Expressing the analyte concentration per mg of creatinine is designed to address this, while also correcting for hydration. The method used for creatinine adjustment also accounts for differences in creatinine excretion related to body size. This does rely on accurate selfreporting of age, height, and weight by the participants, so an estimate of expected creatinine excretion can be made. This may lead to the introduction of inaccuracies due to misreporting of individual characteristics; however, our statistical sensitivity analyses indicated that the DUTCH measurements were in better agreement with the 24-h urine results with the application of the creatinine corrections (see Additional file 1: Figure S4), with the ICC for some metabolites increasing by as much as 0.15 and raising the level of agreement with 24-h measures from good to excellent.
One of the limitations of this 4-spot dried urine method is that reference ranges are laboratory-specific and non-standardized. Still, interpretation of values above and below these reference ranges should be similar to that of other assays. In this study, there was some loss of hormone for estrone and estriol with the filter paper methodology, which may be related to differences in extraction efficiency between the steroid conjugates and creatinine, loss during the drying process, or incomplete saturation of the filter paper. Still, the difference was less than $12 \%$ of the total and would be compensated for by an adjustment of the reference range. In addition, we have previously shown that the dried urine measure of estrone has clinical utility because it is representative of serum estrogen measurements [11]. A number of organic acids plus DHT were more concentrated in the dried urine samples, on average. This may be due to differences in extraction efficiency, a matrix effect or analyte concentration during the drying process; however, this difference did represent less than $10 \%$ of the sample. Fortunately, due to the high level of agreement between the Z-scores from the DUTCH methodology and 24-h collections, laboratory reference ranges should account for these differences. Another issue is there are known genetic differences in glucuronidation of testosterone that may impact relative metabolism and urinary concentrations of testosterone and epitestosterone [29], and this may mean urinary androgen measures are not fully 

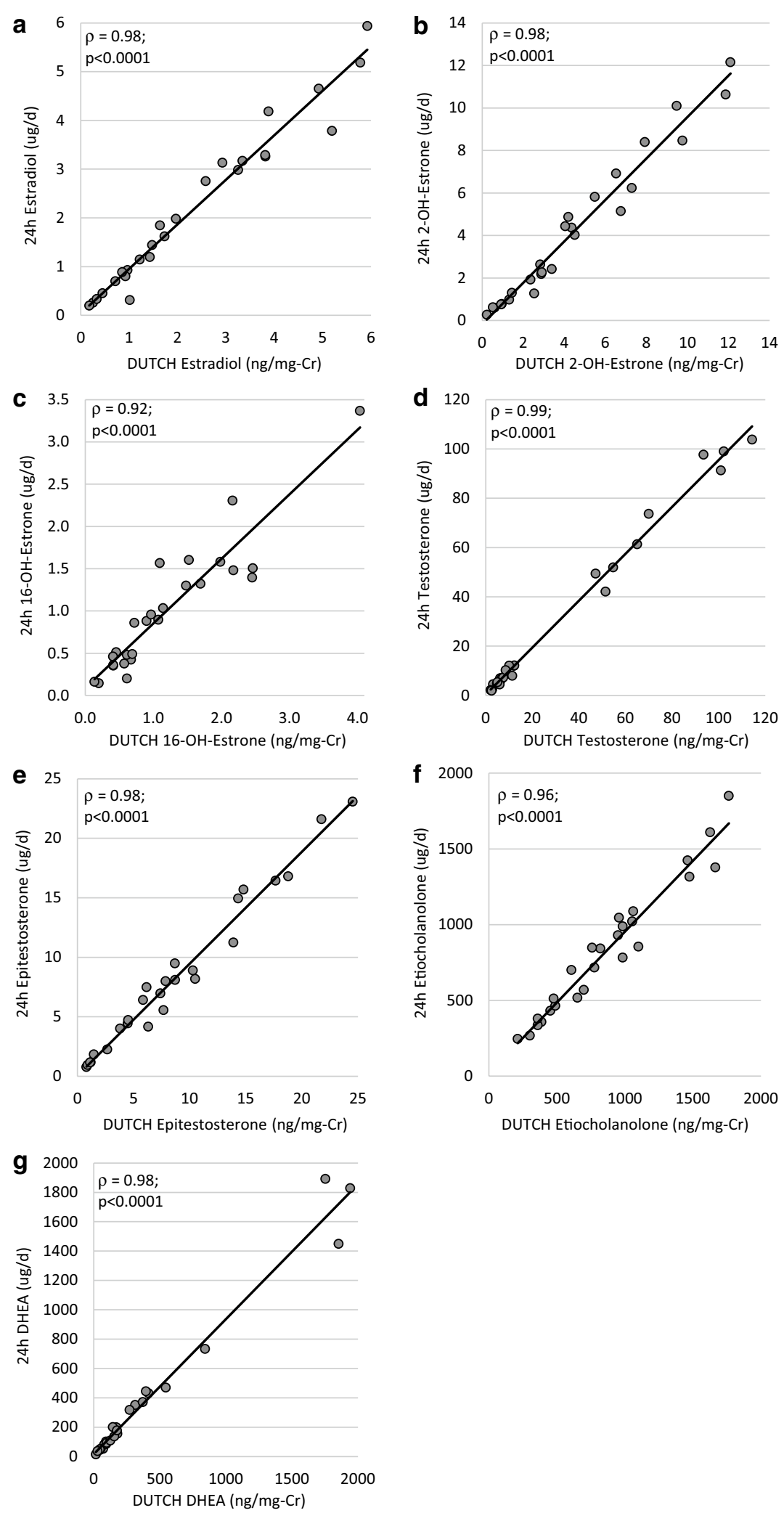

Fig. 3 Correlations between the 24-h urine collection and 4-spot (DUTCH) urine collection measurements. The remainder are available in Additional file 1: Figure S3. Reported correlation coefficients are Spearman correlations. Cr: Creatinine, DHEA: dehydroepiandrosterone, DUTCH: Dried Urine Testing for Comprehensive Hormones 
representative of production rates in a small percentage of individuals. There are also genetic differences in the enzymes that metabolize estrogens, potentially shifting the ratio of 2-hyroxylation to 16-hydroxylation metabolites [30], but these differences may be clinically relevant and indicative of cancer risk [31]. Our study population had limited diversity and was primarily Caucasian, so these results will need to be replicated in more diverse study populations as we could not make inferences regarding any potential differences in results that may have been caused by differences in race.

The methodology of a 4-spot urine collection on filter paper followed by GC-MS/MS or LC-MS/MS offers some advantages. The collection of dried urine on filter paper results in stable measurement of steroid hormones for extended periods of time both by us [11] and others [12] for up to one year [13], even at ambient temperature. Concentrations of organic acids are also stable on dried filter paper for weeks [32, 33]. Mass spectrometry assays, which are now the gold standard for measurement of steroid hormones in blood and urine [10], allow for the use of small sample volumes with excellent sensitivity and accuracy along with simultaneous measurement of a relatively large number of analytes. In combination with chromatography, either gas or liquid, it provides precise separation of closely related molecules [34] by their chemical and physical properties. GC-MS/MS does not exclude any lipophilic steroids, and so a run will contain all excreted steroids [9]. The use of the $H$. pomatia enzymes adds to the accuracy of the quantification of the hormone conjugates, as these enzymes include both a sulfatase and a glucuronidase. The method of GC-MS/ MS does require an additional extraction and derivatization step, but workflows can be optimized to maximize throughput. A 24-h urine collection may be difficult for some patients to fully collect, especially if they are not able to remain at home for an entire day, are disabled, or are incontinent. This methodology removes that barrier and provides the ability to measure multiple hormones at once with a noninvasive collection method, obtaining a complete picture of both production and clearance of the major steroidal hormones.

A multitude of uses, both in research and in clinical scenarios, could be envisioned for assays that are able to measure multiple steroid hormones and organic acids in conveniently collected urine samples on filter paper. For example, the full range of hormonal changes in individuals related to disruption of the natural sleep cycle could be evaluated simultaneously. It is already known that the peak 6-sulfatoxymelatonin, as representative of melatonin, is lower in people working the night shift [35]; a full appreciation of the urinary steroid profile in individuals who work at night could add to this prior research. Similarly, urine profiling may help to fully define the changes expected in genetic syndromes of steroidogenesis $[5,36]$ and errors of metabolism [16]. Dried urine samples may be of particular benefit in screening neonates for organic acid disorders $[15,16]$, and there is recent interest in a possible association of organic acids with neuropsychiatric disorders [37-39]. The ability to look at a full urine profile can provide a more integrated view of the patient; for example, patients using oral contraceptives often have higher xanthurenic acid with concurrent pyroxidine deficiency [40], both of which would be observable using dried urine analysis. A greater understanding of the full effect of changes in hormonal concentrations and metabolites or important clinical subgroups could be determined for both exogenous use of hormones and for exposure to endocrine disrupting compounds like bisphenol A $[41,42]$. Urine hormone profiling might also be used to fully describe age related changes, i.e. through puberty or menopause.

\section{Conclusions}

Mass spectrometry allows for the assessment of a full hormone profile in a small volume of urine such that an expanded view of both hormone production and clearance can be observed. In addition, results from dried urine are in strong agreement with those obtained from liquid urine. In combination with four spot urine collections on filter paper collected throughout the waking hours, we have shown that it is possible to accurately represent a 24-h urine collection. This technology may be useful to the clinician wishing to perform a large series of tests on patients to narrow the differential diagnosis, for those monitoring hormonal therapy or evaluating the menstrual cycle, or for those who need to reduce the burden of collection for their patients. This four-spot, dried urine method allows for assessment of both diurnal patterns [14] as well as total daily production, allowing for a comprehensive evaluation of adrenal and reproductive hormones and other urine metabolites.

\footnotetext{
Abbreviations

2OHE1: 2-Hydroxyestrone; 2-methoxyE1: 2-Methoxyestrone; 4OHE1: 4-Hydroxyestrone; 5-HIAA: 5-Hydroxyindoleacetic acid; 16OHE1: 16-Hydroxyestrone: CAN: Acetonitrile; CLIA: Clinical Laboratory Improvement Amendments; Cr: Creatinine; DHEA: Dehydroepiandrosterone; DHT: $5 \mathbf{a}$-Dihydrotestosterone; DUTCH: Dried Urine Testing for Comprehensive Hormones; E1: Estrone; E2: Estradiol; E3: Estriol; EpiT: Epitestosterone; GC-MS/MS: Gas chromatography with tandem mass spectrometry; Hiv: $\beta$-Hydroxyisovaleric; HVA: Homovanillic acid; ICC: Intraclass correlation coefficient; IQR: Interquartile range; LC-MS/MS: Liquid chromatography with tandem mass spectrometry; MMA: Methylmalonic acid; T: Testosterone; TQD: Tandem quadrupole mass spectrometer detector; VMA: Vanillylmandelic acid.
} 


\section{Supplementary information}

The online version contains supplementary material available at https://doi. org/10.1186/s13065-021-00744-3.

Additional file 1. Table S1: Partial list of urine hormones and analytes that can be measured from dried urine. Figure S1: Correlations between the liquid versus dried measurements for selected urine hormones. Figure S2: Correlations between the liquid versus dried measurements for selected urine organic acids. Figure S3: Correlations between the 24-h urine collection and 4-spot (DUTCH) urine collection measurements. Figure S4: Correlations between the 24-h urine collection and the 4-spot (DUTCH) urine collection estradiol measurements with (C) and without the two creatinine $(\mathrm{Cr})$ corrections $(\mathrm{A} \& \mathrm{~B})$.

\section{Acknowledgements}

The authors would like to acknowledge Danielle Martinot for writing the IRB proposal for this study, Marie Thearle, MD for creating tables and figures and assistance with statistical analysis and interpretation of the data, and Bryan P. Mayfield, PharmD for medical writing assistance. And thanks to the volunteers who provided samples for analysis.

\section{Author's contributions}

MN developed the methodology of the dried urine assay, designed the studies, interpreted the data, and made substantial contributions to the manuscript. DC acquired and interpreted the data and contributed to the preparation of the manuscript. Both authors read and approved the final manuscript.

\section{Funding}

Precision Analytical Laboratory, Inc. provided funds to run urine and serum assays.

\section{Availability of data and materials}

The datasets acquired and/or analyzed during the current study are available from the corresponding author on reasonable request.

\section{Declarations}

\section{Ethics approval and consent to participate}

The research was conducted on healthy adult volunteers at a commercial testing laboratory. Informed consent was obtained from all participants prior to the study or the National University of Natural Medicine Institutional Review Board determined that written informed consent could be waived for the study population. All data reports were deidentified during extraction so the IRB determined that written informed consent could be waived for this study population. Both studies (Clinical Trials ID: NCT04305093) were approved by the National University of Natural Medicine Institutional Review Board. All methods were performed in accordance with the relevant guidelines and regulations required by both the National University of Natural Medicine Institutional Review Board and CLIA.

\section{Consent for publication}

Not applicable.

\section{Competing interests}

Mark Newman is the founder, president, and owner of Preciaion Analytical Inc. Desmond A. Curran is an employee of Precision Analytical. Precision Analytical, Inc. is a commercial laboratory offering hormone testing to medical practitioners and individuals. All statistics were calculated and interpreted by an independent agent.

Received: 4 December 2020 Accepted: 3 March 2021

Published online: 15 March 2021

\section{References}

1. Sansone A, Sansone M, Selleri R et al (2019) Monitoring testosterone replacement therapy with transdermal gel: when and how? J Endocrinol Invest 42(12):1491-1496. https://doi.org/10.1007/s40618-019-01082-x

2. Rodgers M (1997) Adequacy of hormone replacement therapy for osteoporosis prevention assessed by serum oestradiol measurement, and the degree of association with menopausal symptoms. Br J Gen Pract 47(416):161-165

3. de Medeiros SF, Barbosa JS, Yamamoto MM (2015) Comparison of steroidogenic pathways among normoandrogenic and hyperandrogenic polycystic ovary syndrome patients and normal cycling women. J Obstet Gynaecol Res. 41(2):254-263

4. Ketha H, Kaur S, Grebe SK, Singh RJ (2014) Clinical applications of LC-MS sex steroid assays: evolution of methodologies in the 21 st century. Curr Opin Endocrinol Diabetes Obes 21(3):217-226. https://doi.org/10.1097/ MED.0000000000000068

5. Jong WHAD, Buitenwerf E, Pranger AT et al (2017) Determination of reference intervals for urinary steroid profiling using a newly validated GC-MS/MS method. Clin Chem Lab Med 56(1):103-112. https://doi.org/ 10.1515/cclm-2016-1072

6. Nicolau GY, Haus E (1989) Chronobiology of the endocrine system. Endocrinologie. 27(3):153-183

7. Ghiraldi EM, Reddy M, Li T, Lawler AC, Friedlander JI (2017) Factors associated with compliance in submitting 24-hour urine collections in an underserved community. J Endourol 31:S64-S68. https://doi.org/10. 1089/end.2016.0594

8. Sninsky BC, Nakada SY, Penniston KL (2015) Does socioeconomic status, age, or gender influence appointment attendance and completion of 24-hour urine collections? Urology. 85(3):568-573. https://doi.org/10. 1016/j.urology.2014.10.043

9. Krone N, Hughes BA, Lavery GG, Stewart PM, Arlt W, Shackleton CHL (2010) Gas chromatography/mass spectrometry (GC/MS) remains a preeminent discovery tool in clinical steroid investigations even in the era of fast liquid chromatography tandem mass spectrometry (LC/MS/MS). J Steroid Biochem Mol Biol 121(3-5):496-504. https://doi.org/10.1016/j. jsbmb.2010.04.010

10. Stanczyk FZ, Clarke NJ (2010) Advantages and challenges of mass spectrometry assays for steroid hormones. J Steroid Biochem Mol Biol 121(3-5):491-495. https://doi.org/10.1016/j.jsbmb.2010.05.001

11. Newman M, Pratt SM, Curran DA, Stanczyk FZ (2019) Evaluating urinary estrogen and progesterone metabolites using dried filter paper samples and gas chromatography with tandem mass spectrometry (GC-MS/MS). BMC Chem. https://doi.org/10.1186/s13065-019-0539-1

12. Blasco H, Garrigue MA, de Vos A et al (2010) Filter paper saturated by urine sample in metabolic disorders detection by proton magnetic resonance spectroscopy. Anal Bioanal Chem 396(3):1205-1211. https:// doi.org/10.1007/s00216-009-3280-0

13. Shideler SE, Munro CJ, Johl HK, Taylor HW, Lasley BL (1995) Urine and fecal sample collection on filter paper for ovarian hormone evaluations. Am 」 Primatol 37(4):305-315. https://doi.org/10.1002/ajp.1350370405

14. Newman M, Curran DA, Mayfield BP (2020) Dried Urine and Salivary Profiling for Complete Assessment of Cortisol and Cortisol Metabolites. J Clin Trans Endocrinol. https://doi.org/10.1016/j.jcte.2020.100243

15. Fu XW, Iga M, Kimura M, Yamaguchi S (2000) Simplified screening for organic acidemia using GC/MS and dried urine filter paper: a study on neonatal mass screening. Early Human Dev 58(1):41-55. https://doi.org/ 10.1016/S0378-3782(00)00053-0

16. Matsumoto I, Kuhara T (1996) A new chemical diagnostic method for inborn errors of metabolism by mass spectrometry - Rapid, practical, and simultaneous urinary metabolites analysis. Mass Spectrom Rev 15(1):43-57. https://doi.org/10.1002/(SICI)1098-2787(1996)15:1\%3c43: AID-MAS3\%3e3.0.CO;2-B

17. Savkovic S, Lim S, Jayadev V et al (2018) Urine and Serum Sex Steroid Profile in Testosterone-Treated Transgender and Hypogonadal and Healthy Control Men. J Clin Endocrinol Metab 103(6):2277-2283. https://doi.org/ 10.1210/jc.2018-00054 
18. Klein DA, Paradise SL, Reeder RM (2019) Amenorrhea: a systematic approach to diagnosis and management. Am Fam Physician 100(1):39-48

19. Kawasaki T, Uezono K, Itoh K, Ueno M. Prediction of 24-hour urinary creatinine excretion from age, body weight and height of an individual and its application. [Nippon kōshū eisei zasshi] Japanese journal of public health. 1991;38(8):567-574. https://europepmc.org/article/med/1747547. Accessed 5 July 2020

20. Bujang MA, Baharum N (2017) A simplified guide to determination of sample size requirements for estimating the value of intraclass correlation coefficient: a review. 12:1-11

21. Landis JR, Koch GG (1977) The measurement of observer agreement for categorical data. Biometrics 33(1):159. https://doi.org/10.2307/2529310

22. Keenan DM, Veldhuis JD (2016) Pulsatility of hypothalamo-pituitary hormones: a challenge in quantification. Physiology. 31(1):34-50. https:// doi.org/10.1152/physiol.00027.2015

23. Fu X, Kimura M, Iga M, Yamaguchi S (2001) Gas chromatographic-mass spectrometric screening for organic acidemias using dried urine filter paper: determination of a-ketoacids. J Chromatogr B Biomed Sci Appl 758(1):87-94. https://doi.org/10.1016/S0378-4347(01)00101-3

24. Tuchman M, McCann MT, Johnson PE, Lemieux B (1991) Screening newborns for multiple organic acidurias in dried filter paper urine samples: method development. Pediatr Res 30(4):315-321. https://doi.org/10. 1203/00006450-199110000-00005

25. Seviour JA, McGill AC, Craft AW et al (1992) Screening for neuroblastoma in the northern region of england laboratory aspects. J Pediatr Hematol Oncol 14(4):332-336. https://doi.org/10.1097/00043426-19921 1000-00009

26. Tarik M, Ramakrishnan L, Amarchand R et al (2019) Feasibility of measuring sodium, potassium and creatinine from urine sample on dried filter paper. Bioanalysis. 11(8):689-701. https://doi.org/10.4155/bio-2018-0295

27. Monošík R, Dragsted LO (2018) Dried urine swabs as a tool for monitoring metabolite excretion. Bioanalysis. 10(17):1371-1381. https://doi.org/10. 4155/bio-2018-0042

28. Bileck A, Frei S, Vogt B, Groessl M (2020) Urinary steroid profiles: comparison of spot and 24-hour collections. J Steroid Biochem Mol Biol. https:// doi.org/10.1016/j.jsbmb.2020.105662

29. Sten T, Bichlmaier I, Kuuranne T, Leinonen A, Yli-Kauhaluoma J, Finel M (2009) UDP-glucuronosyltransferases (UGTs) 2B7 and UGT2B17 display converse specificity in testosterone and epitestosterone glucuronidation, whereas UGT2A1 conjugates both androgens similarly. Drug Metab Dispos 37(2):417-423. https://doi.org/10.1124/dmd.108.024844

30. Yong M, Schwartz SM, Atkinson C et al (2011) Associations between polymorphisms in glucuronidation and sulfation enzymes and sex steroid concentrations in premenopausal women in the United States. J Steroid Biochem Mol Biol 124(1-2):10-18. https://doi.org/10.1016/j.jsbmb.2010. 12.014

31. Sampson JN, Falk RT, Schairer C et al (2017) Association of estrogen metabolism with breast cancer risk in different cohorts of postmenopausal women. Cancer Res 77(4):918-925. https://doi.org/10. 1158/0008-5472.CAN-16-1717

32. Shinka T, Ohse M, Inoue Y, Kuhara T (2005) Stability of 5-aminolevulinic acid on dried urine filter paper for a diagnostic marker of tyrosinemia type I. J Chromatogr B Analyt Technol Biomed Life Sci 823:44-46

33. McCann M, Thompson M, Gueron IC, Lemieux B, Giguere R, Tuchman M (1996) Methylmalonic acid quantification by stable isotope dilution gas chromatography-mass spectrometry from filter paper urine samples. Clin Chem 42(6 Pt 1):910-914

34. Homer N, Kothiya S, Rutter A, Walker BR, Andrew R (2017) Gas chromatography tandem mass spectrometry offers advantages for urinary steroids analysis. Anal Biochem 538:34-37. https://doi.org/10.1016/j.ab.2017.09. 002

35. Davis S, Mirick DK, Chen C, Stanczyk FZ (2012) Night shift work and hormone levels in women. Cancer Epidemiol Biomark Prev 21(4):609-618. https://doi.org/10.1158/1055-9965.EPI-11-1128

36. Pussard E, Travers S, Bouvattier C et al (2020) Urinary steroidomic profiles by LC-MS/MS to monitor classic 21-Hydroxylase deficiency. J Steroid Biochem Mol Biol. https://doi.org/10.1016/j.jsbmb.2019.105553

37. Marc DT, Ailts JW, Campeau DCA, Bull MJ, Olson KL (2011) Neurotransmitters excreted in the urine as biomarkers of nervous system activity: validity and clinical applicability. Neurosci Biobehav Rev 35(3):635-644. https://doi.org/10.1016/j.neubiorev.2010.07.007

38. Black CN, Bot M, Scheffer PG, Cuijpers P, Penninx BWJH (2015) Is depression associated with increased oxidative stress? A systematic review and meta-analysis. Psychoneuroendocrinology. 51:164-175. https://doi.org/ 10.1016/j.psyneuen.2014.09.025

39. Abou-Saleh MT, Coppen A (1986) Psychiatric progress. The biology of folate in depression: implications for nutritional hypotheses of the psychoses. J Psychiatr Res 20(2):91-101. https://doi.org/10.1016/00223956(86)90009-9

40. Deac OM, Mills JL, Shane B et al (2015) Tryptophan catabolism and vitamin B-6 status are affected by gender and lifestyle factors in healthy young adults. J Nutr 145(4):701-707. https://doi.org/10.3945/jn.114. 203091

41. Kim EJ, Lee D, Chung BC, Pyo H, Lee J (2014) Association between urinary levels of bisphenol-A and estrogen metabolism in Korean adults. Sci Total Environ 470-471:1401-1407. https://doi.org/10.1016/j.scitotenv.2013.07. 040

42. Bloem LM, Storbeck KH, Swart P, du Toit T, Schloms L, Swart AC (2015) Advances in the analytical methodologies: profiling steroids in familiar pathways-challenging dogmas. J Steroid Biochem Mol Biol 153:80-92. https://doi.org/10.1016/j.jsbmb.2015.04.009

\section{Publisher's Note}

Springer Nature remains neutral with regard to jurisdictional claims in published maps and institutional affiliations.
Ready to submit your research? Choose BMC and benefit from:

- fast, convenient online submission

- thorough peer review by experienced researchers in your field

- rapid publication on acceptance

- support for research data, including large and complex data types

- gold Open Access which fosters wider collaboration and increased citations

- maximum visibility for your research: over 100M website views per year

At BMC, research is always in progress.

Learn more biomedcentral.com/submissions 